\title{
CORRECTIONS
}

\section{The Greater Manchester experiment}

Clarification-In the third paragraph of this feature (BMJ 2016;352:i1611, doi:10.1136/bmj.i1611), we should have written that the Taking Charge strategy [for devolution of control of the health and social care budget] was written by the Greater Manchester Combined Authority and the NHS in Manchester. 